\title{
Spatial Distribution of Underweight, Overweight and Obesity among Women and Children: Results from the 2011 Uganda Demographic and Health Survey
}

\author{
Kedir N. Turi ${ }^{1}$, Mary J. Christoph ${ }^{1}$ and Diana S. Grigsby-Toussaint ${ }^{1,2, *}$
}

1 Department of Kinesiology and Community Health, University of Illinois at Urbana-Champaign, 1206 S. Fourth Street, 80B Huff Hall, Champaign, IL 61820, USA;

E-Mails: knturi@illinois.edu (K.N.T.); mchrstp2@illinois.edu (M.J.C.)

2 Division of Nutritional Sciences, University of Illinois at Urbana-Champaign, 1206 S. Fourth Street, 2019 Huff Hall, Champaign, IL 61820, USA

* Author to whom correspondence should be addressed; E-Mail: dgrigs1@illinois.edu; Tel: +1-217-333-2307; Fax: +1-217-333-2766.

Received: 31 July 2013; in revised form: 27 September 2013 / Accepted: 29 September 2013 / Published: 11 October 2013

\begin{abstract}
While undernutrition and infectious diseases are still persistent in developing countries, overweight, obesity, and associated comorbidities have become more prevalent. Uganda, a developing sub-Saharan African country, is currently experiencing the public health paradox of undernutrition and overnutrition. We utilized the 2011 Uganda Demographic and Health Survey (DHS) to examine risk factors and hot spots for underweight, overweight, and obesity among adult females $(N=2,420)$ and their children $(N=1,099)$ using ordinary least squares and multinomial logit regression and the ArcGIS Getis-Ord Gi* statistic. Overweight and obese women were significantly more likely to have overweight children, and overweight was correlated with being in the highest wealth class $(\mathrm{OR}=2.94,95 \% \mathrm{CI}=1.99-4.35)$, and residing in an urban $(\mathrm{OR}=1.76,95 \% \mathrm{CI}=1.34-2.29)$ but not a conflict prone $(\mathrm{OR}=0.48,95 \% \mathrm{CI}=0.29-0.78)$ area. Underweight clustered significantly in the Northern and Northeastern regions, while overweight females and children clustered in the Southeast. We demonstrate that the DHS can be used to assess geographic clustering and burden of disease, thereby allowing for targeted programs and policies. Further, we pinpoint specific regions and population groups in Uganda for targeted preventive measures and treatment to reduce the burden of overweight and chronic diseases in Uganda.
\end{abstract}


Keywords: obesity; Uganda; sub-Saharan Africa; spatial epidemiology; geographic information systems

\section{Introduction}

As many developing countries undergo economic, epidemiologic, and nutrition transitions, the coexistence of undernutrition and overnutrition has emerged as a public health paradox [1]. While undernutrition and infectious diseases persist, increased levels of overweight, obesity, and associated comorbidities have accompanied the nutrition transition in developing nations, and now cause a significant burden of disease [2]. Increased rates of overweight and obesity, as well as associated chronic diseases such as hypertension and diabetes, have been recently observed in many developing countries [2-4]. Noncommunicable diseases account for over $60 \%$ of all global deaths [5], with $80 \%$ now occurring in low- or middle-income countries [6]. One region undergoing rapid and sweeping changes in terms of demography, nutrition, and health is sub-Saharan Africa [7,8]. Overweight and obesity in the region increased 35\% between 1992 and 2005 [8], and at least one study has shown that hypertension quadrupled from 2005 to 2008 to an overall prevalence of $16.2 \%$ [9]. Additionally, Hall et al. showed that diabetes prevalence varied from $1 \%$ to $12 \%$ of the population for parts of the region, costing an estimated $\$ 8,836$ per patient or a total of $\$ 67.03$ billion per year [10].

Understanding the geographic distribution of obesity is integral to designing interventions that target high-risk areas. Although spatial clustering and other geographic information systems (GIS) techniques are commonly employed to study the patterns and distribution of infectious and chronic diseases [11,12], most studies examining geographical differences in risk factors surrounding obesity have been limited to developed countries [13], including characteristics such as high traffic and poverty in Quebec [14], homicide rates and green space in New York City [15], and aspects of the nutrition and physical activity environments in the northwest US [16]. Other studies have correlated risk factors for obesity, such as physical inactivity to neighborhood green space [17] and walkability [18].

In one of the first studies analyzing the geographic distribution of overweight in a developing country, and in particular sub-Saharan Africa, Pawloski et al. [19] used longitudinal Demographic and Health Survey data to conclude that overweight and obesity had increased in Kenya from 2003 to 2009, and showed through a Getis-Ord Gi* analysis that overweight clustered in mothers and their children. Another analysis in northeast China found that combined overweight and obesity prevalence in urban children and adolescents was high (33\% for males and $18 \%$ for females), whereas prevalence was intermediate in wealthy rural areas and still very low in impoverished rural areas [20]. While these studies both identified geographic areas with high obesity, they linked hotspots for overweight with few contributing risk factors, thereby limiting our understanding of the overall implications for designing policies and interventions to encourage healthy weight. We address this gap by using GIS techniques to identify hot spots for underweight and overweight, and correlating geographic data with ordinary least squares (OLS) and multinomial logistic regression of sociodemographic risk factors including age, marital status, education, income, rural/urban residence, and contextualizing this within population-based risk factors including proximity to refugee settlements and cash crop production. 
We chose Uganda as a representative low-income country in sub-Saharan Africa because of its diverse geography and ethnic groups, relative political stability, and availability of recent geographic and statistical data from the 2011 Demographic Health Survey. Overweight and obesity, as well as associated chronic diseases such as hypertension [9] and diabetes [21], are quickly becoming more prevalent in Uganda [21-24]. The national prevalence of overweight and obesity among adult females ages $15-49$ is $19 \%$, but areas such as the capital, Kampala, have much higher levels, with $40.4 \%$ of women being overweight and 13\% being obese [24]. Women are more likely to be overweight than men [21]; a small study in eastern Uganda revealed that overweight was associated with being female, increased age, high socioeconomic status, and peri-urban residence [21]. Even as overweight is increasing, malnutrition persists in Uganda. The prevalence of underweight in children under 5 actually increased from $28 \%$ in 2004-2006 to 38\% in 2010-2012 [25], and iron deficiency is projected to cause as many as 15,000 maternal deaths by 2015 [26].

In addition, Uganda was also selected for this analysis because the authors are currently conducting pilot studies to identify and target interventions for obesity based on the Analysis Grid for Environments Linked to Obesity (ANGELO) framework developed by Swinburn et al. [27]. This framework was developed to focus on microenvironmental settings (e.g., neighborhood food stores) and macroenvironmental sectors (e.g., health care systems) that act as preventive or aggravating factors to obesity risk. The current analysis will help us to better target areas for future studies and the engagement of stakeholders in Uganda. Previous studies analyzing the risk factors for overweight and obesity in Uganda have failed to account for the vast diversity in the agricultural landscape, socioeconomic factors, and political stability [28]. Our statistical and geospatial analyses show that publicly available datasets such as the DHS can be used to assess the burden of underweight and overweight and associated risk factors - both geographically and by sociodemographic characteristics - and thus provide a basis for targeted policies and interventions.

\section{Experimental Section}

\subsection{Data Source}

We utilized publicly available data from the 2011 Uganda Demographic and Health Survey (DHS). These health surveys are nationally representative, population-based surveys that provide indicators of demographics, health, and nutrition [24]. This comprehensive household survey (9,033 households in 404 enumeration areas) included a questionnaire targeted to women. The Woman's Questionnaire asked eligible women ages 15-49 in each of the households about their background, work, birth history, fertility preferences, breastfeeding, and vaccinations, in addition to other health indicators. The survey was conducted at a multistage cluster level with each cluster comprising 5-10 women per set of geographic coordinates [24]. Each observation includes information about the target female of reproductive age in each household, up to six children, and household characteristics.

The data originally consisted of a sample of 8,674 rural and urban women and 2,336 children under age 5. However, after exclusions, the sample consisted of 2,420 adult females and 1,099 child observations. We excluded observations that lacked height and weight for adults (5,912 cases) and height, weight, age, and sex for children (1,237 cases). We excluded adults with extreme body mass 
index values (BMI > 80; 54 cases), and pregnant women (288 cases). We also excluded children younger than 6 months of age who were likely breastfeeding, because childhood overweight and obesity generally develop after the introduction of supplemental foods [29]. The majority of infants in Uganda are not exclusively breastfed after 6 months, so we included children ages 6 months to 5 years [30]. GIS coordinate data (latitude and longitude) from the 2011 DHS database were then merged with demographic data for our final sample. Based on a comparison with excluded cases, our final sample was representative both geographically and demographically of the entire sample. All regions across Uganda were represented by approximately $30 \%$ of the original DHS sample with BMI data for mothers and children [24].

\subsection{Anthropometric Measures}

Adults were classified as overweight or obese according to the World Health Organization (WHO) definition [3], with a BMI of less than $18.5 \mathrm{~kg} / \mathrm{m}^{2}$ classified as underweight, 18.5 to $25 \mathrm{~kg} / \mathrm{m}^{2}$ comprising normal weight, greater than $25 \mathrm{~kg} / \mathrm{m}^{2}$ overweight, and over $30 \mathrm{~kg} / \mathrm{m}^{2}$ being obese. Overweight for children was classified according to the 2006 WHO Child Growth Standards as being two standard deviations (+2 SD) above the median weight-for-height based on the child's specific age and sex [31].

\subsection{Explanatory Variables}

Explanatory variables were chosen based on basic characteristics and established correlations with obesity $[19,23,24]$. The marital status of adult women was recategorized as ever married and never married. The ever married category included divorced, separated, widowed and currently married adult women. Age was defined as age of women in years at the time of the survey. Mothers' education level was recorded as number of years women spent in school, which ranged from 0 to 18 years of education. It was included in the regression analysis as a continuous variable. The residence of adult women was recorded as rural or urban based on the Ugandan census [32]. A wealth index constructed using household assets and services data via principal components analysis by the administrators of the DHS was divided into five quintiles: very poor, poor, medium, rich and very rich. For simplicity of analysis we combined very poor and poor together to form the low wealth category, rich and very rich to form the high wealth category and the medium quintile to form the medium wealth category. In order to provide context for the wider ecological, economic and political factors that may be impacting underweight and overweight risk, we also created regional measures of political instability and agricultural production. Using data on humanitarian response programs [33] and major cash crop farming [34], we categorized regions as being conflict prone based on the presence of refugee camps and settlements, or as cash crop producing based on the presence of major coffee, cotton, or tea production.

\subsection{Statistical Analysis}

We used both OLS and multinomial logistic regression to examine demographic and socioeconomic factors that contributed to weight status for mothers and their children. OLS regression has advantages over the categorical estimation methods because it uses all the data points during the estimation. 
However, it does not provide the probability of moving from normal weight to underweight or from normal weight to overweight or obese as an effect of each risk factor. Therefore, in addition to OLS, we used multinomial logistic regression to estimate the shift in risk of mothers' weight status due to underlying socioeconomic and demographic risk factors. The regression analysis was performed using Stata-SE version 12.0 (Statacorp, College Station, TX, USA, 2011).

\subsection{Spatial Analysis}

We used ArcGIS version 10.1 (ESRI, Redlands, CA, USA, 2011) to investigate the clustering of underweight and overweight or obesity. Spatial analysis was performed at the cluster level, composed of an average of 5 to 10 aggregated households. The areas with DHS sample clusters did not necessarily overlap with administrative boundaries. Rather, these clusters were GPS coordinate readings taken at the nearest town or community center for an artificially designated area for survey by DHS. Using this point data, we performed Getis-Ord Gi* analyses [35] for local clusters in order to measure global and local clustering of BMI for mothers and to calculate weight-height-age percentiles for children and residual risk factors for mothers and children. Getis-Ord Gi* [35] was an appropriate analytical method for detecting hot spots (and cold spots) for underweight and overweight mothers and children because we have point data based on latitude and longitude coordinates at the center of DHS clusters of sample households.

As previously discussed, the unit of spatial analysis is a cluster of sample households as designated by DHS. In order to prevent the exclusion of local factors by imposing sharp boundaries, the concept of the "zone of indifference" was used for the distance a hot spot boundary covers. Using this method, the neighboring sample households within the specified critical distance boundary of a target DHS sample-household cluster are included in analyses for designating the cluster as a hot spot (or cold spot). Once the specified critical distance boundary is reached, the level of impact of the neighboring clusters is reduced [36]. The computation of GiZ score for local Getis-Ord Gi* [35] is given as:

$$
G_{i}^{*}(d)=\frac{\left(\sum_{j=1}^{n} w_{i j} x_{j}-\bar{X} \sum_{j=1}^{n} w_{i j}\right)}{S \sqrt{\frac{\left[n \sum_{j=1}^{n} w_{i j}^{2}-\left(\sum_{j=1}^{n} w_{i j}\right)^{2}\right]}{n-1}}}
$$

where $\bar{X}=\frac{\sum_{j=1}^{n} x_{j}}{n}$ and $S=\sqrt{\frac{\sum_{j=1}^{n} x_{j}^{2}}{n}}-(\bar{X})^{2} i$ and $j$ are geographical locations (coordinates) and $x_{i}$ and $x_{j}$ are values of the variables under study (BMI for adults and BMI percentile for children) at the coordinates $i$ and $j$. The variable $w_{i j}$ stands for the association weights for coordinates $i$ and $j$ at distance $d$.

In contrast to measures of global spatial autocorrelation, which return a single-value $z$ score for each variable (indicating the degree of regional clustering across the entire distribution of that variable), measures of local spatial autocorrelation produce one value for each location for each variable, indicating the degree to which that particular location is part of a hot spot or cold spot [37].

The local Getis-Ord Gi* statistic compares the local mean rate (the mean of the rates for a cluster of DHS sample households and its nearest neighboring cluster of DHS sample households) to the global mean rate (the mean of the rates for all clusters of DHS sample households). It produces a $z$ score and a $p$-value for each cluster of DHS sample households, reflecting whether the differences between the local 
and global means are statistically significant or not. A statistically significant positive $z$ score indicates a hot spot of high rates, meaning that the values are so abnormally high that the chances are very low that the spatial clustering results from random processes. Similarly, a statistically significant negative $z$ score for a DHS sample-household cluster indicates local clustering of especially low rates, or a cold spot $[35,38]$. We used a 95\% statistical significance level, which corresponds to $z$ scores $>1.96$ and $<-1.96$ as cut-off points for significant hot spots and cold spots, respectively.

\section{Results and Discussion}

\subsection{Risk Analysis}

Table 1 summarizes sociodemographic characteristics and weight status of adult women and children in our sample. Out of the 2,420 women, $19 \%$ were overweight or obese. Eight percent $(n=195)$ of the women were underweight, $73 \%(n=1,756)$ were normal weight, $15 \%(n=363)$ were overweight, and $4 \%(n=106)$ were obese (data not shown). Urban residents were more likely to be overweight or obese $(32 \%)$ than rural residents $(13 \%)$. Overweight was significantly correlated with wealth: only $6 \%$ of women in the lowest wealth class were overweight or obese, whereas $30 \%$ of women in the wealthiest class were overweight or obese. Education followed the same trend: $13 \%$ of women with no education were overweight, whereas overweight increased to $16 \%$ with primary level education, to $25 \%$ with secondary level education, and to $41 \%$ with above secondary level education. Overweight or obese women were also more likely to have overweight children (19\%) compared to their normal-weight or underweight counterparts (14\%) (data not shown).

Table 1. Sociodemographic characteristics and weight status of the sample of adult females $(N=2,420)$ and children $(N=1,099)$. Statistically significant differences were observed for all sociodemographic characteristics between normal/underweight and overweight/obese groups; *An asterisk indicates a statistically significant trend $(p<0.001)$.

\begin{tabular}{lcccc}
\hline Variables & Normal/Underweight & $\mathbf{\%}$ & Overweight/Obese & $\mathbf{\%}$ \\
\hline $\begin{array}{l}\text { Sample population } \\
\text { Residence }\end{array}$ & 1,951 & 81 & 469 & 19 \\
$\quad$ Urban & 522 & 68 & 248 & 32 \\
$\quad$ Rural & 1,429 & 87 & 221 & 13 \\
Wealth class * & & & & \\
Low & 780 & 94 & 53 & 6 \\
Medium & 335 & 87 & 51 & 13 \\
High & 836 & 70 & 365 & 30 \\
Education * & & & & \\
None & 291 & 87 & 45 & 13 \\
Primary & 1,131 & 84 & 217 & 16 \\
Secondary & 435 & 75 & 141 & 25 \\
Above secondary & 94 & 59 & 66 & 41 \\
Children's BMI percentile category & 951 & 86 & 148 & 13 \\
\hline
\end{tabular}


Table 2. Ordinary least square regression analysis relating socioeconomic and demographic risk factors for maternal and child weight. Reference categories are never married for marital status, no education for maternal education level, low wealth for wealth class, non-cash crop producing region, and non-conflict prone region. $\mathrm{SE}=$ standard error. ** $p<0.05, * * * p<0.001$.

\begin{tabular}{|c|c|c|c|c|}
\hline \multirow{2}{*}{ Variables } & \multicolumn{4}{|c|}{ Coefficients } \\
\hline & Mothers $(N=\mathbf{2 , 4 2 0})$ & SE & Children $(N=1,099)$ & SE \\
\hline Maternal BMI & & & $0.015 * * *$ & 0.004 \\
\hline Age & $0.044 * * *$ & 0.010 & $0.038 * *$ & 0.012 \\
\hline Marital status: ever married & $1.002 * * *$ & 0.21 & & \\
\hline Residence: urban & $1.079 * * *$ & 0.19 & & \\
\hline Maternal education level & $0.054 * * *$ & 0.022 & -0.003 & 0.003 \\
\hline Wealth class: medium & $0.75 * * *$ & 0.24 & & \\
\hline Wealth class: $h i g h$ & $1.61 * * *$ & 0.23 & & \\
\hline Cash crop producing region: yes & 0.75 & 0.18 & & \\
\hline Conflict prone region: yes & -0.81 & 0.23 & & \\
\hline Intercept & $18.61 * * *$ & 0.31 & -0.003 & 0.003 \\
\hline $\mathrm{R}^{2}$ & $18.17 \%$ & & $4.52 \%$ & \\
\hline
\end{tabular}

Multinomial logit regression showed that being underweight was significantly and negatively associated with being wealthy or ever married, whereas overweight was significantly and positively associated with increasing age, high wealth status, and residence in an urban area (Table 3). The odds of being overweight increased to 2.94 as a woman's wealth status increased from low to high (Table 3). Residence in a conflict-prone region was protective $(\mathrm{OR}=0.48)$ against overweight/obesity risk (Table 3$)$.

Table 3. Multinomial regression results for the association between socioeconomic and demographic risk factors and weight status of adult women in the sample $(N=2,420)$. References for the risk factors are never married for marital status, rural for residence, no education for education level, and low wealth for wealth class, non-cash crop producing region, and non-conflict prone region. The odds ratios use normal weight status as the reference. Normal weight is the denominator. $\mathrm{SE}=$ standard error; $\mathrm{CI}=$ confidence interval. $* p<0.1, * * p<0.05, * * * p<0.001$.

\begin{tabular}{|c|c|c|c|c|}
\hline \multirow{2}{*}{ Variables } & \multicolumn{2}{|c|}{ Underweight } & \multicolumn{2}{|c|}{ Overweight and obese } \\
\hline & Log odds ratio (SE) & Odds ratio $[95 \% \mathrm{CI}]$ & Log odds ratio (SE) & Odds ratio $[95 \% \mathrm{CI}]$ \\
\hline Age & $0.022(0.011)$ & $1.022[1.000,1.045]$ & $0.044 * * *(0.0075)$ & $1.045 * * *[1.030,1.052]$ \\
\hline $\begin{array}{l}\text { Marital status: ever } \\
\text { married }\end{array}$ & $-0.66 * *(0.25)$ & $0.512 * *[0.32,0.84]$ & $0.36 *(0.17)$ & $1.43 *[1.034,1.98]$ \\
\hline Residence: urban & $0.0029(0.27)$ & $1.003[0.59,1.69]$ & $0.56 * * *(0.14)$ & $1.76 * * *[1.34,2.29]$ \\
\hline Education level & $-0.028(0.028)$ & $0.97[0.92,1.027]$ & $0.031 *(0.016)$ & $1.032 *[1.001,1.063]$ \\
\hline Wealth class: medium & $-0.89 * *(0.27)$ & $0.41 * *[0.24,0.69]$ & $0.34(0.23)$ & $1.41[0.89,2.19]$ \\
\hline Wealth class: high & $-0.92 * * *(0.26)$ & $0.39 * * *[0.24,0.66]$ & $1.079 * * *(0.19)$ & $2.94 * * *[1.99,4.35]$ \\
\hline $\begin{array}{l}\text { Cash crop producing } \\
\text { region: yes }\end{array}$ & $-0.32(0.22)$ & $0.72[0.47,1.11]$ & $0.23(0.13)$ & $1.26[0.97,1.62]$ \\
\hline Conflict prone region: yes & $0.17(0.22)$ & $1.19[0.78,1.81]$ & $-0.73 * *(0.24)$ & $0.48 * *[0.29,0.78]$ \\
\hline Intercept & $-1.72 * * *(0.35)$ & & $-3.98 * * *(0.28)$ & \\
\hline
\end{tabular}




\subsection{Spatial Analysis Results}

Figure 1 displays the percentage of overweight and obese mothers in Uganda by district, ranging from $0 \%$ to almost $64 \%$ overweight in some regions. Regions in the Southwest are considerably overrepresented here, with the districts including Kampala, the capital, and Mbarara, the third largest city in Uganda, having two of the highest rates of overweight and obesity, ranging from 19\% to $63 \%$.

Figure 1. The percentage of overweight mothers in Uganda shown as quintiles, by district, where darker districts experience higher levels of overweight and obesity.

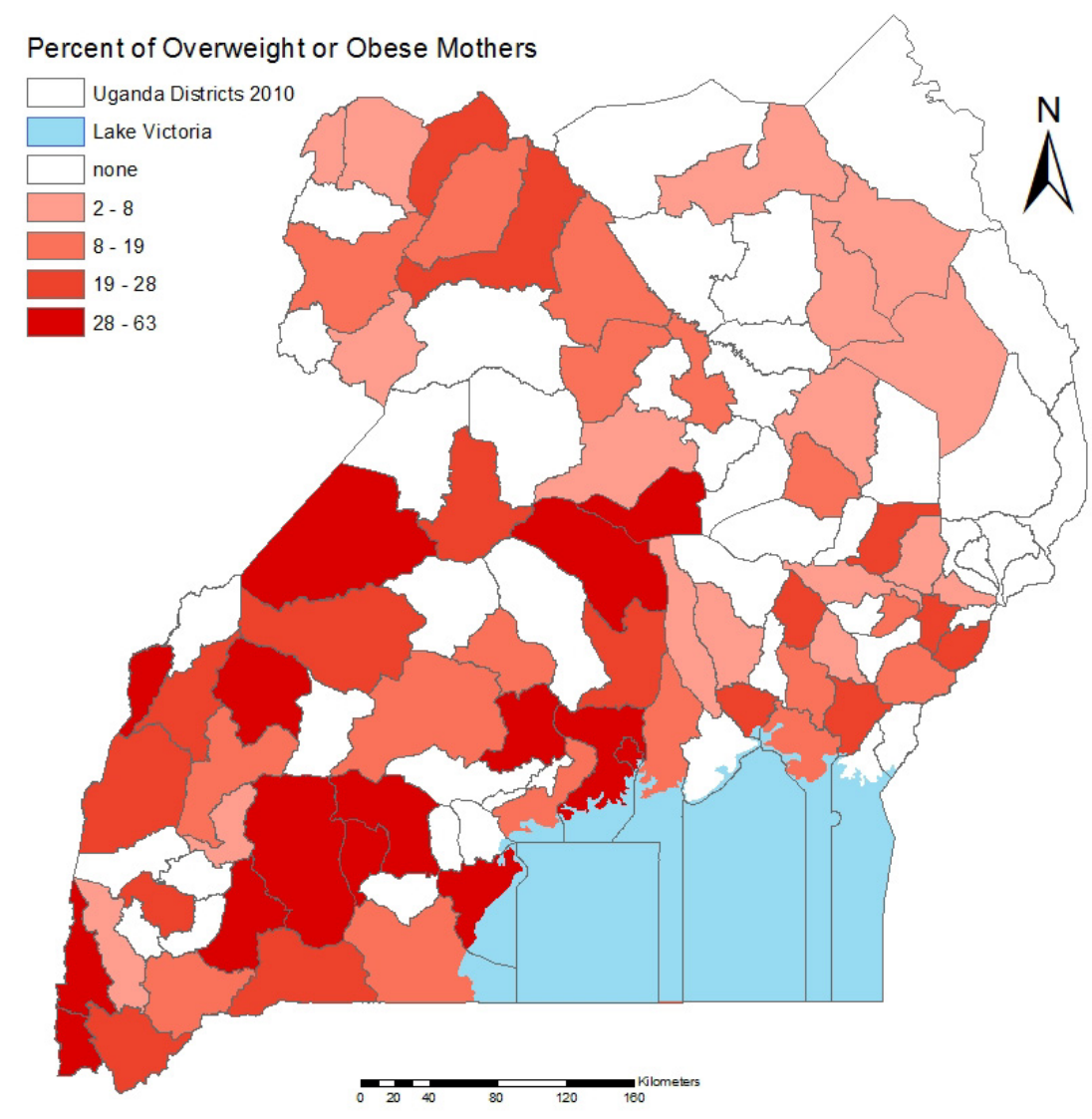

Figure 2 shows the percentage of underweight mothers by district, ranging from $0 \%$ to $34 \%$. Not surprisingly, the distribution is almost completely the reverse of the overweight map. The Karamoja region in the northeast has an extremely high prevalence of underweight (15-34\% region-wide), and the east central region is also over-represented.

Figure 3 shows the Getis-Ord Gi* Hot Spot analysis for overweight and obese women in Uganda. This shows a striking trend, with the entire southern region facing a significant burden of overweight and obesity among adult women, while the northern regions suffer from underweight. All but one of the regions with the highest clustering of overweight are in a major city; these are the major cities of Kampala, Mbarara, Masaka and a smaller city, Kabale [32]. 
Figure 2. The percentage of underweight mothers in Uganda as quintiles, by district, where darker districts experience higher levels of underweight.

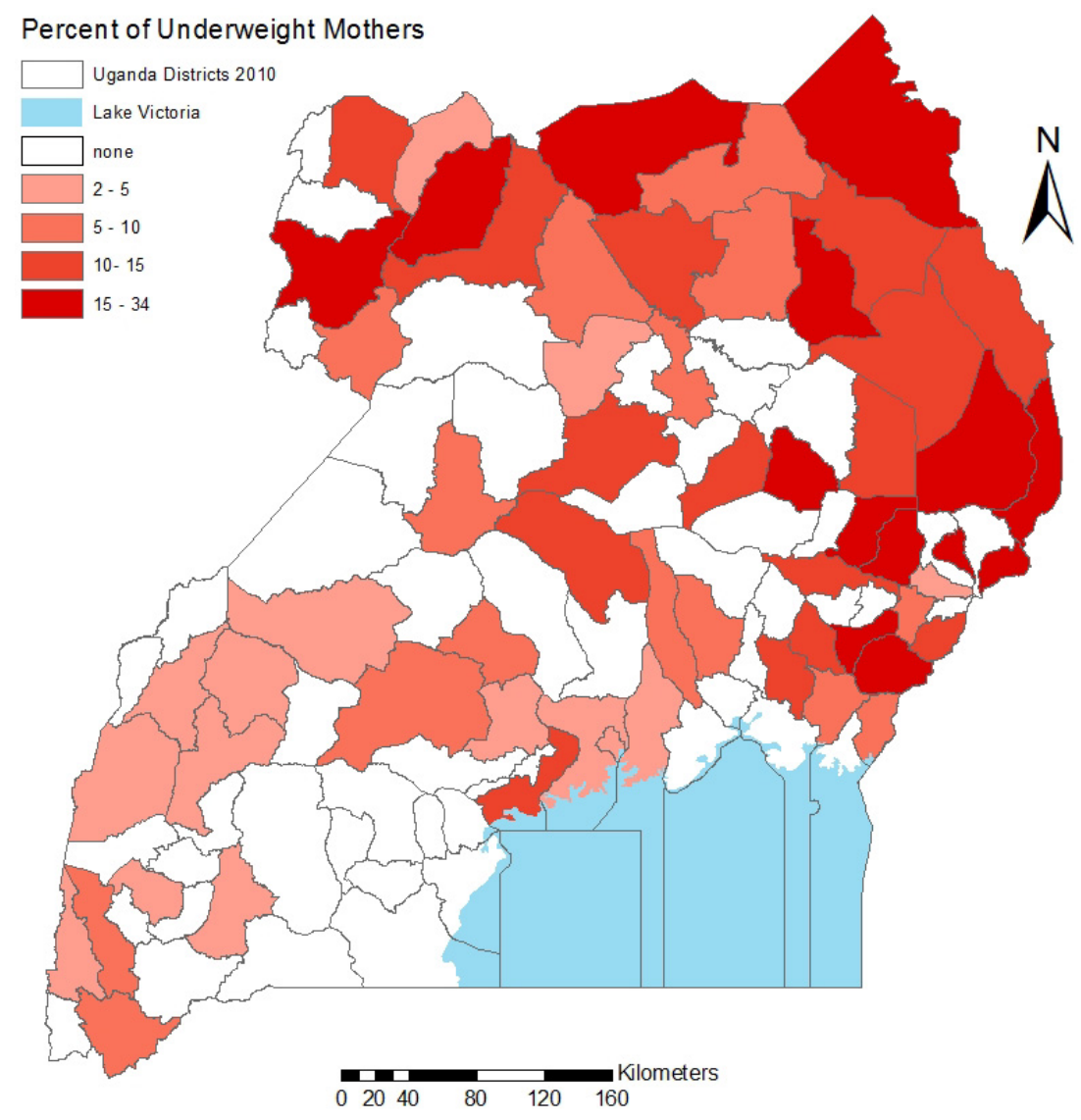

As noted in the experimental section, BMI for children is calculated differently than BMI for adults, with children at or above the 85th percentile for weight, height, and age being considered overweight. The Getis-Ord Gi* Hot Spot analysis of clusters in children (Figure 4) shows that the prevalence of both overweight $(16.70 \%)$ and underweight $(6 \%)$ is much lower in children than in their mothers, but that many of the hot spots for overweight children are found in the regions where the greatest amount of maternal overweight is observed. Again, overweight is clustered in the Kampala and Southwest districts.

\subsection{Discussion}

Our results suggest that there is a significant burden of overweight in Uganda in urban areas and in wealthier regions of the country. Overweight and obesity are correlated with higher age, living in an urban area, and high wealth. The Southwest region and the capital city of Kampala had the highest rates of obesity, with the rate of overweight reaching $40 \%$. The prevalence of overweight in children was much lower overall (17\%), but is still significant in Kampala, several major cities in the southwestern corner, and two major northern cities. Overweight children were more likely than their normal-weight or underweight counterparts to have overweight or obese mothers. However, in the area surrounding the major northern city of Lira, two significant hot spots emerged for children even though no such significant hot spots occurred for their mothers. This validates the results of Pawloski et al. in Kenya, in which most hot spots emerged for both mothers and children, even though some hot spots for overweight 
in children occurred with normal-weight mothers, and some hot spots for mothers occurred with normal-weight children [19].

Figure 3. Hot and cold spots of maternal BMI. Dark red dots represent hot spots of high maternal BMI, and dark blue dots represent cold spots of low maternal BMI at the $95 \%$ statistical significance level. Each dot represents a cluster of DHS sample households, while the boundaries shown are districts. Dots inside Lake Victoria are located on islands.

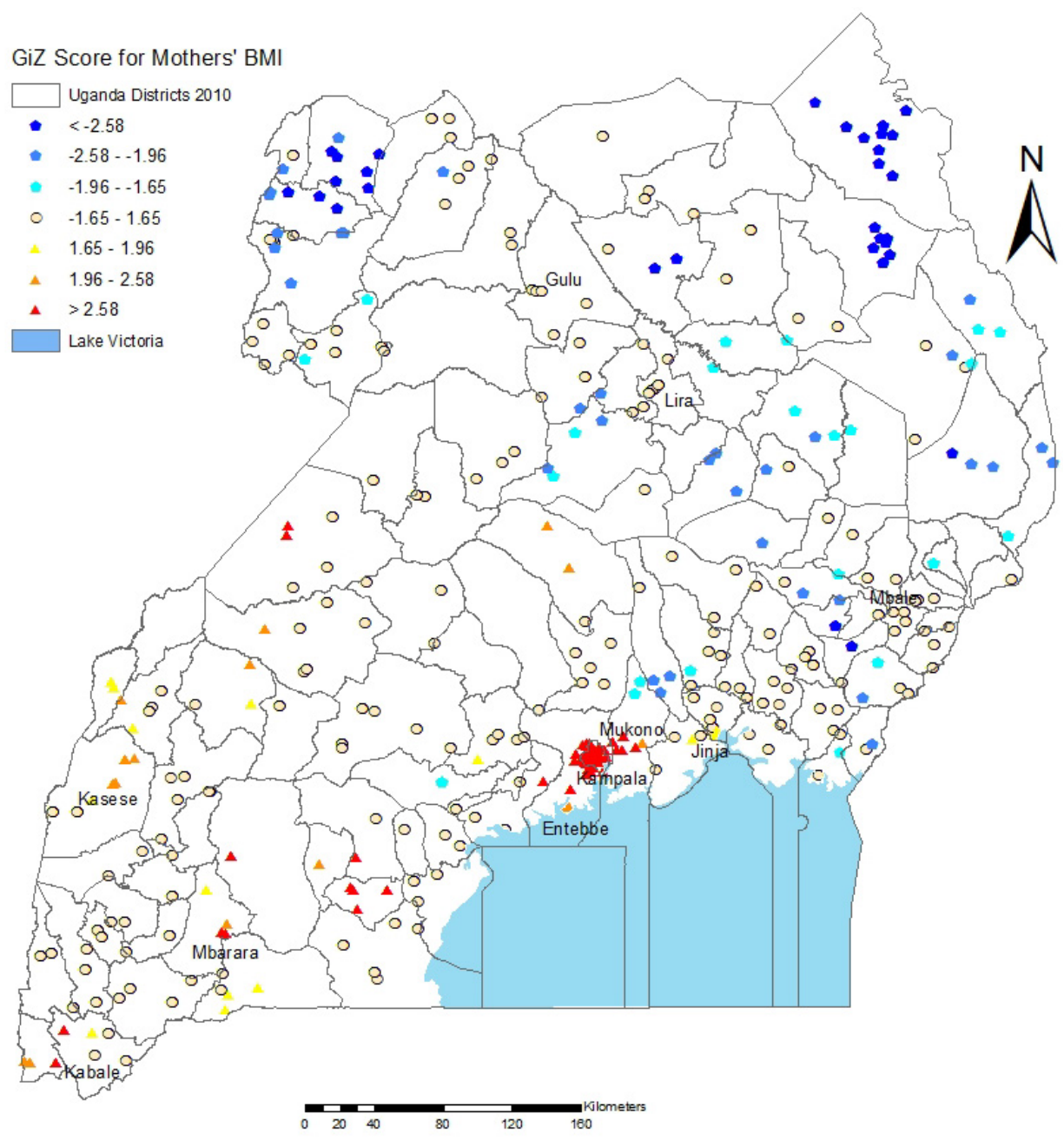

The prevalence of overweight and obesity was decidedly lower in the North. Even though overweight was associated with urban residence, the three largest cities in the North (Gulu, Lira, and Mbale) did not emerge as hot spots for overweight and obesity in mothers or children. This is likely related to the high levels of displacement and poverty accompanying the 21-year civil war in the North between the Ugandan government and rebel groups, including the Lord's Resistance Army [39-41], which is borne out in our analysis. The Karamoja region in the far Northeast is one of the most malnourished in the country, and the North suffers from the highest level of food insecurity [28], possibly explaining some of the variability within the country.

While the issues affecting obesity risk are complex, the Southwest likely has a high prevalence of overweight because of its relative political stability, greater presence of tourism and nongovernmental organizations that provide economic support and supplemental nutrition, increased economic 
opportunities, higher rainfall and cash crop yields, and subsequent higher level of food security. Furthermore, these factors have led to better education and higher wealth, which also correlate with higher weight status in Uganda [24,28].

Figure 4. Hot and cold spots of child BMI percentile. Dark red dots represent hot spots of high child BMI percentile, and dark blue dots represent cold spots of low child BMI percentile at the $95 \%$ statistical significance level. Each dot represents a cluster of DHS sample households, while the boundaries shown are districts. Dots inside Lake Victoria are located on islands.

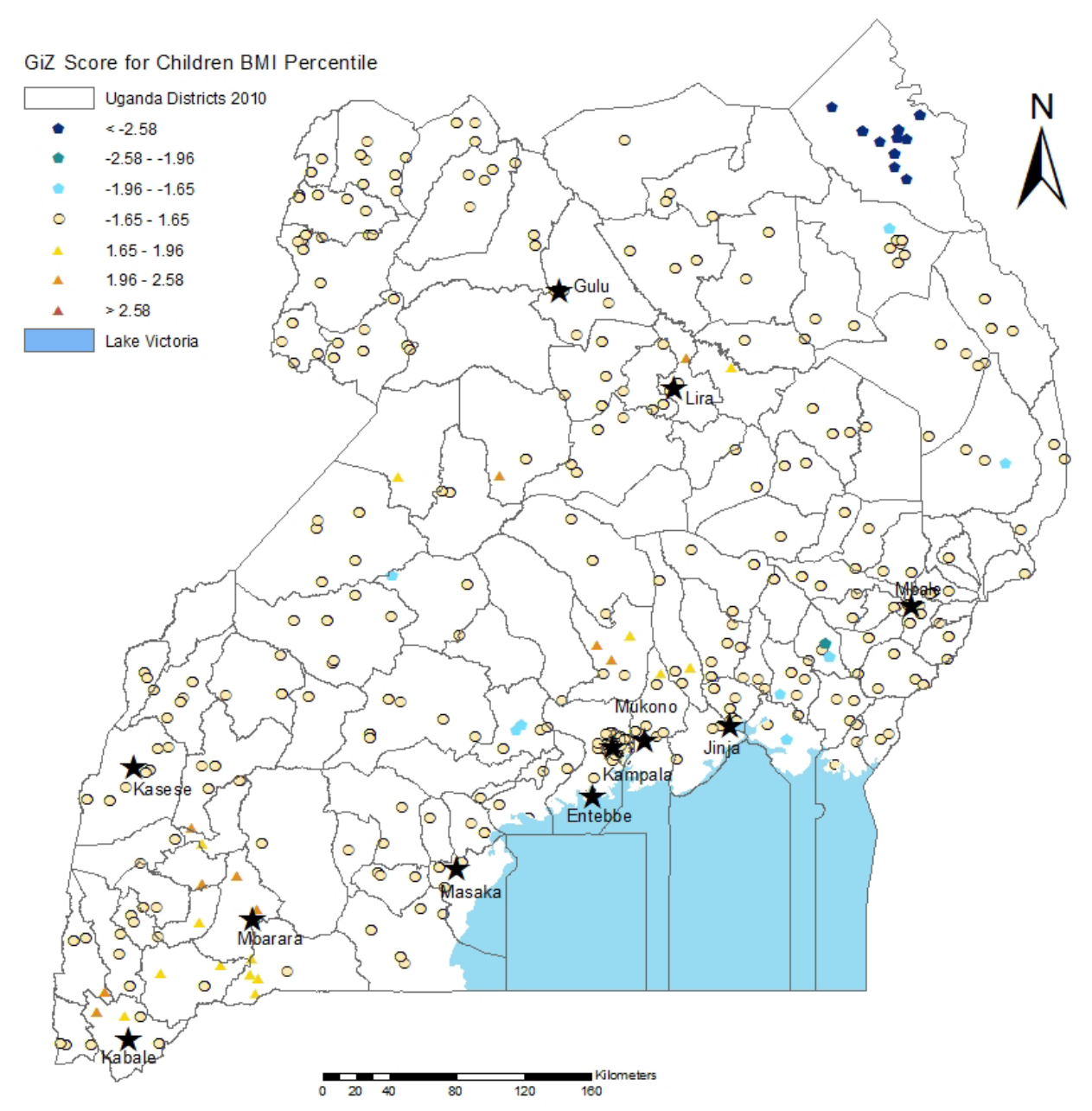

\subsection{Strengths and Limitations}

Our study provides insights into the demographic and geographic correlates of weight status in Uganda. Despite the limitations associated with utilizing the DHS for our analysis (described below), this survey remains the largest nationally representative dataset including weight status coupled with geographic coordinates for Uganda. Moreover, van Wesenbeeck, Keyzer, and Nube have validated the use of the DHS to survey nutrition-related health outcomes in Africa [42].

Our study has several limitations. First, a substantial subset of adult women from our initial sample was excluded due to the lack of anthropometric measurements necessary for calculating BMI. Exclusions notwithstanding, after comparing our final sample with the excluded cases, we found that our final sample was representative both geographically and demographically of the entire sample. Second, 
the DHS does not include items on health behaviors that are related to obesity risk, such as daily physical activity levels, which makes it challenging to draw inferences about other individual-level factors that may lead to overweight or obesity. Third, aside from young children and females of child-bearing age, specific population groups are not well represented, although the 2011 DHS did survey adult males. Fourth, the DHS aggregates groups of 5-10 women into single sets of coordinates, thereby limiting the exact point data. However, since this was a countrywide study with small cluster areas that have very similar environmental characteristics, this point-data limitation should not affect the conclusions drawn from our study.

\section{Conclusions}

Our study is one of the first spatial analyses of overweight and obesity in Uganda. It is currently one of the few and most in-depth focused on a low-income country, accounting for both individual- and broader socio-political factors age such as age and internal displacement that may influence population-based differences in weight status. We used GIS techniques to show regional variation in maternal-child overweight and obesity in Uganda. Our analysis illustrates how geospatial and statistical tools can be used with publicly available datasets - such as the DHS - to elucidate some of the risk factors and sociodemographic characteristics related to overweight and obesity. In so doing, we show that the DHS can be better utilized as a tool for tracking the long-term implications of undernutrition and overnutrition in Uganda and sub-Saharan Africa.

Consequently, our work underscores the growing value of using geostatistical tools to elucidate the etiology of overweight and obesity and the increasing rates of chronic disease in sub-Saharan Africa. Future research should focus on how regionally specific environmental factors may affect the risk of overweight and obesity. Collection of more in-depth data concerning other risk factors for chronic disease, such as physical activity levels, is necessary for a comprehensive understanding of the development and pathology of obesity in sub-Saharan Africa. The DHS and other nationally representative surveys that provide spatial coordinates can be utilized for determining spatial clusters and correlates for overweight and obesity that may not be obvious using traditional regression methods, and can provide a starting point for developing tailored interventions and policies to address the impact of noncommunicable diseases in Uganda and sub-Saharan Africa.

\section{Acknowledgments}

Kedir N. Turi is supported by research funds for Diana Grigsby-Toussaint from the Department of Kinesiology and Community Health at the University of Illinois. Mary J. Christoph is supported by the National Institute for Agriculture under the Illinois Transdisciplinary Obesity Prevention Program (ITOPP) training grant (2011-67001-30101) to the Division of Nutritional Sciences at the University of Illinois. We acknowledge Marilyn O'hara's spatial epidemiology course at the University of Illinois for helping to inspire this work. We thank our reviewers for a meticulous reading of our manuscript to enhance the presentation of our research. We thank the U.S. Agency for International Development, ICF International, and the Uganda Bureau of Statistics for making the data from the DHS available. 


\section{Conflicts of Interest}

The authors declare no conflict of interest.

\section{References}

1. Tanumihardjo, S.A.; Anderson, C.; Kaufer-Horwitz, M.; Bode, L.; Emenaker, N.J.; Haqq, A.M.; Satia, J.A.; Silver, H.J.; Stadler, D.D. Poverty, obesity, and malnutrition: An international perspective recognizing the paradox. J. Am. Diet. Assoc. 2007, 107, 1966-1972.

2. Popkin, B.M.; Adair, L.S.; Ng, S.W. Global nutrition transition and the pandemic of obesity in developing countries. Nutr. Rev. 2012, 70, 3-21.

3. World Health Organization. Global InfoBase. Available online: https://apps.who.int/infobase/ (accessed on 22 October 2012).

4. Finucane, M.M.; Stevens, G.A.; Cowan, M.J.; Danaei, G.; Lin, J.K.; Paciorek, C.J.; Singh, G.M.; Gutierrez, H.R.; Lu, Y.; Bahalim, A.N.; et al. National, regional, and global trends in body-mass index since 1980: Systematic analysis of health examination surveys and epidemiological studies with 960 country-years and 9.1 million participants. Lancet 2011, 377, 557-567.

5. Bloom, D.E.; Cafiero, E.T.; Jané-Llopis, E.; Abrahams-Gessel, S.; Bloom, L.R.; Fathima, S.; Feigl, A.B.; Gaziano, T.; Mowafi, M.; Pandya, A.; et al. The Global Economic Burden of Noncommunicable Diseases; World Economic Forum: Geneva, Switzerland, 2011.

6. World Health Organization (WHO). Global Status Report on Noncommunicable Diseases 2010; WHO: Geneva, Switzerland, 2011.

7. Dalal, S.; Beunza, J.J.; Volmink, J.; Adebamowo, C.; Bajunirwe, F.; Njelekela, M.; Mozaffarian, D.; Fawzi, W.; Willett, W.; Adami, H.O.; et al. Non-Communicable diseases in sub-Saharan Africa: What we know now. Int. J. Epidemiol. 2011, 40, 885-901.

8. Ziraba, A.K.; Fotso, J.C.; Ochako, R. Overweight and obesity in urban Africa: A problem of the rich or the poor? BMC Public Health 2009, 9, 465, doi:10.1186/1471-2458-9-465.

9. Twagirumukiza, M.; de Bacquer, D.; Kips, J.G.; de Backer, G.; Stichele, R.V.; van Bortel, L.M. Current and projected prevalence of arterial hypertension in sub-Saharan Africa by sex, age and habitat: An estimate from population studies. J. Hypertens 2011, 29, 1243-1252.

10. Hall, V.; Thomsen, R.W.; Henriksen, O.; Lohse, N. Diabetes in sub-Saharan Africa 1999-2011: Epidemiology and public health implications. A systematic review. BMC Public Health 2011, 11, doi:10.1186/1471-2458-11-564.

11. Cromley, E.K. GIS and disease. Annu. Rev. Publ. Health 2003, 24, 7-24.

12. Rushton, G. Public health, GIS, and spatial analytic tools. Ann. Rev. Publ. Health 2003, 24, 43-56.

13. Pearce, J.; Witten, K.; Bartie, P. Neighbourhoods and health: A GIS approach to measuring community resource accessibility. J. Epidemiol. Commun. Health 2006, 60, 389-395.

14. Van Hulst, A.; Gauvin, L.; Kestens, Y.; Barnett, T.A. Neighborhood built and social environment characteristics: A multilevel analysis of associations with obesity among children and their parents. Int. J. Obes. (Lond.) 2013, doi:10.1038/ijo.2013.81. 
15. Lovasi, G.S.; Schwartz-Soicher, O.; Quinn, J.W.; Berger, D.K.; Neckerman, K.M.; Jaslow, R.; Lee, K.K.; Rundle, A. Neighborhood safety and green space as predictors of obesity among preschool children from low-income families in New York City. Prev. Med. 2013, 57, 189-193.

16. Saelens, B.E.; Sallis, J.F.; Frank, L.D.; Couch, S.C.; Zhou, C.; Colburn, T.; Cain, K.L.; Chapman, J.; Glanz, K. Obesogenic neighborhood environments, child and parent obesity: The neighborhood impact on kids study. Am. J. Prev. Med. 2012, 42, e57-e64.

17. Grigsby-Toussaint, D.S.; Chi, S.H.; Fiese, B.H. Where they live, how they play: Neighborhood greenness and outdoor physical activity among preschoolers. Int. J. Health Geogr. 2011, 10, doi:10.1186/1476-072X-10-66.

18. Laxer, R.E.; Janssen, I. The proportion of youths' physical inactivity attributable to neighbourhood built environment features. Int. J. Health Geogr. 2013, 12, 31, doi:10.1186/1476-072X-12-31.

19. Pawloski, L.R.; Curtin, K.M.; Gewa, C.; Attaway, D. Maternal-child overweight/obesity and undernutrition in Kenya: A geographic analysis. Public Health Nutr. 2012, 15, 2140-2147.

20. Ji, C.Y.; Cheng, T.O. Prevalence and geographic distribution of childhood obesity in China in 2005. Int. J. Cardiol. 2008, 131, 1-8.

21. Lasky, D.; Becerra, E.; Boto, W.; Otim, M.; Ntambi, J. Obesity and gender differences in the risk of type 2 diabetes mellitus in Uganda. Nutrition 2002, 18, 417-421.

22. Mayega, R.W.; Makumbi, F.; Rutebemberwa, E.; Peterson, S.; Ostenson, C.G.; Tomson, G.; Guwatudde, D. Modifiable socio-behavioural factors associated with overweight and hypertension among persons aged 35 to 60 years in eastern Uganda. PLoS One 2012, 7, doi:10.1371/journal.pone.0047632.

23. Maher, D.; Waswa, L.; Baisley, K.; Karabarinde, A.; Unwin, N.; Grosskurth, H. Distribution of hyperglycaemia and related cardiovascular disease risk factors in low-income countries: A cross-sectional population-based survey in rural Uganda. Int. J. Epidemiol. 2011, 40, 160-171.

24. Uganda Bureau of Statistics (UBOS). ICF International Inc. Uganda Demographic and Health Survey 2011; UBOS: Kampala, Uganda, and ICF International Inc.: Calverton, MD, USA, 2012; pp. 143-162.

25. Food and Agriculture Organization. FAOSTAT: Country Profiles: Uganda. Available online: http://faostat.fao.org/site/666/default.aspx (accessed on 30 May 2013).

26. Uganda PROFILES. Nutrition Advocacy Technical Working Group PROFILES Meeting Report; Ministry of Health: Kampala, Uganda, 2010.

27. Swinburn, B.; Egger, G.; Raza, F. Dissecting obesogenic environments: The development and application of a framework for identifying and prioritizing environmental interventions for obesity. Prev. Med. 1999, 29, 563-570.

28. FANTA-2. The Analysis of the Nutrition Situation in Uganda; Food and Nutrition Technical Assistance II Project (FANTA-2); FHI 360: Washington, DC, USA, 2010.

29. Wilson, A.C.; Forsyth, J.S.; Greene, S.A.; Irvine, L.; Hau, C.; Howie, P.W. Relation of infant diet to childhood health: Seven year follow up of cohort of children in Dundee infant feeding study. Brit. Med. J. 1998, 316, 21-25.

30. Kikafunda, J.K.; Walker, A.F.; Tumwine, J.K. Weaning foods and practices in central Uganda: A cross-sectional study. Afr. J. Food Agr. Nutr. Dev. 2003, 3. Available online: http://www.bioline.org.br/request?nd03012\#kikafunda (accessed on 30 July 2013). 
31. Who Child Growth Standards: Length/Height-for-Age, Weight-for-Age, Weight-for-Length, Weight-for-Height and Body Mass Index-for-Age: Methods and Development; WHO: Geneva, Switzerland, 2006.

32. Uganda Bureau of Statistics. The 2002 Uganda Population and Housing Census: Analytical Report; UBOS: Kampala, Uganda, 2006. Available online: http://www.ubos.org/onlinefiles/ uploads/ubos/pdf documents/2002 CensusPopnSizeGrowthAnalyticalReport.pdf (accessed on 1 July 2013).

33. Humanitarian Response Common Operational Datasets (COD)-Fundamental Operational Datasets (FODs) registry. Uganda: Admin Level 3 Boundaries and Settlements. Available online: http://cod.humanitarianresponse.info/fr/search/field_country_region/187?search_api_views_fullte xt (accessed on 3 September 2013).

34. Geographic Information Support Team (GIST). Repository at the University of Georgia. Uganda Cash Crop Production. Available online: https://gistdata.itos.uga.edu/ (accessed on 3 September 2013).

35. Ord, J.K.; Getis, A. Local spatial autocorrelation statistics: Distributional issues and an application. Geogr. Anal. 1995, 27, 286-306.

36. Mitchell, A. The ESRI Guide to GIS Analysis; ESRI Press: Redlands, CA, USA, 2005; Volume 2.

37. Grieve, J. A Comparison of Statistical Methods for the Aggregation of Regional Linguistic Variation. In Aggregating Dialectology and Typology: Linguistic Variation in Text and Speech, Within and Across Languages; Szmrecsanyi, B., Walchli, B., Eds.; Walter de Gruyter: Berlin, Germany, 2013.

38. Getis, A.; Ord, J.K. The analysis of spatial association by use of distance statistics. Geogr. Anal. 1992, 24, 189-206.

39. Behrend, H. Alice Lakwena and the Holy Spirits: War in Northern Uganda 1985-97; Fountain Publishers: Kampala, Uganda, 1999.

40. Finnström, S. Living with Bad Surroundings: War and Existential Uncertainty in Acholiland., Northern Uganda; Uppsala University Press: Uppsala, Sweden, 2003.

41. Branch, A. Neither peace nor justice: Political violence and the peasantry in northern Uganda, 1986-1998. Afr. Stud. Quart 2005, 8. Available online: http://web.africa.ufl.edu/asq/v8/ v8i2a1.htm (accessed on 30 August 2013).

42. Van Wesenbeeck, C.F.; Keyzer, M.A.; Nube, M. Estimation of undernutrition and mean calorie intake in Africa: Methodology, findings and implications. Int. J. Health Geogr. 2009, 8, doi:10.1186/1476-072X-8-37.

(C) 2013 by the authors; licensee MDPI, Basel, Switzerland. This article is an open access article distributed under the terms and conditions of the Creative Commons Attribution license (http://creativecommons.org/licenses/by/3.0/). 